\title{
Research Article Sufficient Optimality and Sensitivity Analysis of a Parameterized Min-Max Programming
}

\author{
Huijuan Xiong, ${ }^{1}$ Yu Xiao, ${ }^{2}$ and Chaohong Song ${ }^{1}$ \\ ${ }^{1}$ College of Science, Huazhong Agricultural University, Wuhan 430070, China \\ ${ }^{2}$ School of Basic Science, East China Jiaotong University, Nanchang 330000, China \\ Correspondence should be addressed to Huijuan Xiong, xiongdou1231@gmail.com
}

Received 4 June 2012; Accepted 17 July 2012

Academic Editor: Jian-Wen Peng

Copyright (C) 2012 Huijuan Xiong et al. This is an open access article distributed under the Creative Commons Attribution License, which permits unrestricted use, distribution, and reproduction in any medium, provided the original work is properly cited.

Sufficient optimality and sensitivity of a parameterized min-max programming with fixed feasible set are analyzed. Based on Clarke's subdifferential and Chaney's second-order directional derivative, sufficient optimality of the parameterized min-max programming is discussed first. Moreover, under a convex assumption on the objective function, a subdifferential computation formula of the marginal function is obtained. The assumptions are satisfied naturally for some application problems. Moreover, the formulae based on these assumptions are concise and convenient for algorithmic purpose to solve the applications.

\section{Introduction}

In this paper, sufficient optimality and sensitivity analysis of a parameterized min-max programming are given. The paper is triggered by a local reduction algorithmic strategy for solving following nonsmooth semi-infinite min-max-min programming $\left(\mathrm{SIM}^{3} \mathrm{P}\right.$, see $[1,2]$, etc. for related applications reference):

$$
\begin{array}{ll}
\min _{x} & f(x) \\
\text { s.t. } & g(x)=\max _{y \in Y} \min _{1 \leq i \leq q}\left\{g_{i}(x, y)\right\} \leq 0 .
\end{array}
$$


With the local reduction technique, the $\mathrm{SIM}^{3} \mathrm{P}$ can be rewritten as a bilevel programming first, where the lower problem is the following parameterized min-max programming $P_{x}$ (see [35] for related reference of local reduction strategy):

$$
\begin{array}{ll}
\min _{y} & g(x, y)=\max _{1 \leq i \leq q}\left\{-g_{i}(x, y)\right\} \\
\text { s.t. } & y \in Y .
\end{array}
$$

To make the bilevel strategy applicable to $\mathrm{SIM}^{3} \mathrm{P}$, it is essential to discuss the second-order sufficient optimality of $P_{x}$ and give sensitivity analysis of the parameterized minimum $y(x)$ and corresponding marginal function $g(x, y(x))$.

Sensitivity analysis of optimization problems is an important aspect in the field of operation and optimization research. Based on different assumptions, many results on kinds of parametric programming have been obtained ([6-9], etc.). Among these, some conclusions on parameterized min-max programming like (1.2) have also been given. For example, based on variation analysis, parameterized continuous programming with fixed constraint was discussed in [7]. Problem like (1.2) can be seen as a special case. Under the inf-compactness condition and the condition objective function is concave with respect to the parameter, directional derivative computational formula of marginal function for (1.2) can be obtained directly. However, concave condition cannot be satisfied for many problems. Recently, Fréchet subgradients computation formula of marginal functions for nondifferentiable programming in Asplund spaces was given ([9]). By using Fréchet subgradients computation formula in [9], subgradient formula of marginal function for (1.2) is direct. But the formula is tedious, if utilizing the formula to construct optimality system of (1.1), the system is so complex that it is difficult to solve the obtained optimality system.

For more convenient computational purpose, the focus of this paper is to establish sufficient optimality and simple computation formula of marginal function for (1.2). Based on Clarke's subdifferential and Chaney's second-order directional derivative, sufficient optimality of the parameterized programming $P_{x}$ is given first. And then Lipschitzian continuousness of the parameterized isolated minimizer $y(x)$ and the marginal function $g(y(x), x)$ is discussed; moreover, subdifferential computation formula of the marginal function is obtained.

\section{Main Results}

Let $Y$ in (1.2) be defined as $Y=\left\{y \in R^{m}: h_{i}(y) \leq 0, i=1, \ldots, l\right\}$, where $h_{i}(\cdot)$ and $i=1, \ldots, l$, are twice continuously differentiable functions on $R^{m}$, and $g_{i}(\cdot, \cdot)$ in (1.2) are twice continuously differentiable functions on $R^{n \times m}$. In the following, we first give the sufficient optimality condition of (1.2) based on Clarke's subdifferential and Chaney's second-order directional derivative, and then make sensitivity analysis of the parameterized problem $P_{x}$. 


\subsection{Sufficient Optimality Conditions of $P_{x}$}

Definition 2.1 (see [10]). For a given parameter $x$, a point $y^{*} \in Y$ is said to be an local minimum of problem $P_{x}$ if there exists a neighborhood $U$ of $y^{*}$ such that

$$
g(x, y) \geq g\left(x, y^{*}\right), \quad \forall y \in U \cap Y, y \neq y^{*}
$$

Assumption 2.2. For a given parameter $x$, suppose that $P_{x}$ satisfying the following constraint qualification:

$$
\left\{d \in R^{m}: \nabla h_{i}(y)^{T} d<0, \forall i \in I_{h}(y), y \in Y\right\} \neq \emptyset
$$

where $I_{h}(y)=\left\{i=\{1, \ldots, l\}: h_{i}(y)=0\right\}$.

For a given parameter $x$, denote the Lagrange function of $P_{x}$ as $L(x, y, \lambda)=g(x, y)+$ $\sum_{i=1}^{l} \lambda_{i} h_{i}(y)$, then the following holds.

Theorem 2.3. For a given parameter $x$, if $y^{*}$ is a minimum of $P_{x}$, Assumption 2.2 holds, then there exists a $\lambda^{*} \in R_{+}^{l}$ such that $0 \in \partial_{y} L\left(x, y^{*}, \lambda\right)$, where $\partial_{y} L\left(x, y^{*}, \lambda^{*}\right)$ denotes the Clarke's subdifferential of $L\left(x, y^{*}, \lambda^{*}\right)$. Specifically, the following system holds:

$$
0 \in \partial_{y} g\left(x, y^{*}\right)+\sum_{i=1}^{l} \lambda_{i} \nabla h_{i}\left(y^{*}\right)
$$

where $\partial_{y} g(x, y)$ denotes Clarke's subdifferential of $g(x, y)$ with respect to $y$, it can be computed as $\operatorname{co}\left\{\nabla_{y} g_{i}\left(x, y^{*}\right): i \in I\left(x, y^{*}\right)\right\}, \operatorname{co}\{\cdot\}$ is an operation of making convex hull of the elements, $I\left(x, y^{*}\right)=\left\{i \in\{1, \ldots, q\}: g\left(x, y^{*}\right)=g\left(x, y^{*}\right)\right\}$.

Proof. The conclusion is direct from Theorem 3.2.6 and Corollary 5.1.8 in [11].

Since $g(x, y)=\max _{1 \leq i \leq p}\left\{g_{i}(x, y)\right\}$ is a directional differentiable function (Theorem 3.2.13 in [11]), the directional derivative of $g(x, y)$ with respect to $y$ in direction $d$ can be computed as follows:

$$
g_{y}^{\prime}(x, y ; d)=\max \left\{\xi^{T} d: \forall \xi \in \partial_{y} g(x, y), \forall d \in R^{m}\right\}
$$

Definition 2.4 (see [10]). Let $f(x)$ is a locally Lipschitzian function on $R^{n}, u$ be a nonzero vector in $R^{n}$. Suppose that

$$
d \in \partial_{u} f(x)=\left\{v \in R^{n}: \exists\left\{x_{k}\right\},\left\{v_{k}\right\}, \text { s. t. } x_{k} \stackrel{x}{\longrightarrow}, v_{k} \longrightarrow v, v_{k} \in \partial f\left(x_{k}\right) \text { for each } k\right\}
$$

define Chaney's lower second-order directional derivative as follows:

$$
f_{-}^{\prime \prime}(x, v, u)=\liminf \frac{f\left(x_{k}\right)-f(x)-v^{T}\left(x_{k}-x\right)}{t_{k}^{2}}
$$


taking over all triples of sequences $\left\{x_{k}\right\},\left\{v_{k}\right\}$, and $\left\{t_{k}\right\}$ for which

(a) $t_{k}>0$ for each $k$ and $\left\{x_{k}\right\} \rightarrow x$;

(b) $t_{k} \rightarrow 0$ and $\left(x_{k}-x^{*}\right) / t_{k}$ converges to $u$;

(c) $\left\{v_{k}\right\} \rightarrow v$ with $v_{k} \in \partial f\left(x_{k}\right)$ for each $k$.

Similarly, Chaney's upper second-order directional derivative can be defined as

$$
f_{+}^{\prime \prime}=\limsup \frac{f\left(x_{k}\right)-f(x)-v^{T}\left(x_{k}-x\right)}{t_{k}^{2}}
$$

taking over all triples of sequences $\left\{x_{k}\right\},\left\{v_{k}\right\}$, and $\left\{t_{k}\right\}$ for which (a), (b), and (c) above hold.

For parameterized max-type function $g(x, y)=\max _{1 \leq i \leq p}\left\{-g_{i}(x, y)\right\}$, where $x$ is a given parameter, its Chaney's lower and upper second-order directional derivatives can be computed as follows.

Proposition 2.5 (see [12]). For any given parameter $x$, Chaney's lower and upper second-order directional derivatives of $g(x, y)$ with respect to $y$ exist; moreover, for any given $0 \neq u \in R^{q}, v \in$ $\partial_{u} g(x, y)$, it has

$$
\begin{aligned}
& g_{-}^{\prime \prime}(x, y ; d)=\min \left\{\frac{1}{2} \sum_{i=1}^{q} a_{j} u^{T} \nabla_{y}^{2} g_{i}(x, y) u: a \in T_{u}(g, y, v)\right\}, \\
& g_{+}^{\prime \prime}(x, y ; d)=\max \left\{\frac{1}{2} \sum_{i=1}^{q} a_{j} u^{T} \nabla_{y}^{2} g_{i}(x, y) u: a \in T_{u}(g, y, v)\right\},
\end{aligned}
$$

where

$$
T_{u}(g, y, v)=\left\{\begin{aligned}
& \exists\left\{y^{(k)}\right\},\left\{a^{(k)}\right\},\left\{v^{(k)}\right\}, \text { such that } \\
&(1) y^{(k)} \longrightarrow y \text { in direction } u, \\
& a \in R_{+}^{q}:\text { (2) } \left.v^{(k)} \longrightarrow v, \text { and } v^{(k)} \in \partial_{y} g\left(x, y^{(k)}\right), k=1,2, \ldots,\right\}, \\
& \text { (3) } a^{(k)} \longrightarrow a, a^{(k)} \in E_{q}, v^{(k)}=\sum_{i=1}^{p} a_{i}^{(k)} \nabla_{y} g_{i}\left(x, y^{(k)}\right), \\
& \text { (4) } a_{j}^{(k)}=0, \text { for } j \notin K_{g}\left(y^{(k)}\right)
\end{aligned}\right\}
$$

where $K_{g}\left(y^{(k)}\right)=\left\{i \in Q: g_{i}\left(x, y^{(n)}\right)=g\left(x, y^{(n)}\right), \exists y^{(n)} \in B(y, 1 / n), \forall n \in N\right\}, E_{q}=\left\{a \in R_{+}^{q}\right.$ : $\left.\sum_{i=1}^{p} a_{i}=1\right\}, Q=\{1, \ldots, q\}$, and $B(y, 1 / n)$ denotes the ball centered in $y$ with radius $1 / n$.

Theorem 2.6 (sufficiency theorem). For a given parameter $x \in R^{n}$, Assumption 2.2 holds, then there exists $y^{*} \in R^{m}$ such that (2.3) holds. Moreover, for any feasible direction $d \in R^{m}$ of $Y$, that is, $\max \left\{\nabla h_{i}\left(y^{*}\right)^{T} d: 1 \leq i \leq l\right\} \leq 0$, if d satisfying one of the following conditions: 
Journal of Applied Mathematics

(1) $g_{y}^{\prime}\left(x, y^{*} ; d\right) \neq 0$;

(2) $g_{y}^{\prime}\left(x, y^{*} ; d\right)=0, \sum_{i=1}^{l} \lambda_{i} \nabla h_{i}(y)^{T} d=0$, that is, $L_{y}^{\prime}(x, y ; d)=0$, and

$$
\min \left\{\frac{1}{2} \sum_{i=1}^{q} a_{i} d^{T} \nabla_{y}^{2} g_{i}\left(x, y^{*}\right) d: a \in E_{q}\right\}+\sum_{i=1}^{l} \lambda_{i} d^{T} \nabla^{2} h_{i}\left(y^{*}\right) d>0,
$$

then $y^{*}$ is a local minimum of $P_{x}$.

Proof. (1) If not, then there exists sequences $t_{k} \downarrow 0, d_{k} \rightarrow d, y_{k}=y^{*}+t_{k} d_{k} \in Y$ such that

$$
g\left(x, y_{k}\right)<g\left(x, y^{*}\right)
$$

As a result, $g_{y}^{\prime}\left(x, y^{*} ; d\right)=\lim _{t \downarrow 0}\left(g\left(x, y^{*}+t d\right)-g\left(x, y^{*}\right)\right) / t=\lim _{k \rightarrow+\infty}\left(g\left(x, y^{*}+t_{k} d_{k}\right)-\right.$ $\left.g\left(x, y^{*}\right)\right) / t_{k} \leq 0$. If $g_{y}^{\prime}\left(x, y^{*} ; d\right) \neq 0$, then $g_{y}^{\prime}\left(x, y^{*} ; d\right)<0$. From (2.4), we know that $\xi^{T} d<$ 0 for all $\xi \in \partial_{y} g\left(x, y^{*}\right)$. Hence, for the direction $d \in R^{m}$, we have

$$
\xi^{T} d+\sum_{i=1}^{l} \nabla h_{i}\left(y^{*}\right)^{T} d<0, \quad \xi \in \partial_{y} g\left(x, y^{*}\right)
$$

On the other hand, from $y^{*}$ satisfying (2.3), we know that there exists a $\xi \in \partial_{y} g\left(x, y^{*}\right)$ such that

$$
\xi^{T} d+\sum_{i=1}^{l} \nabla h_{i}\left(y^{*}\right)^{T} d=0
$$

which leads to a contradiction to (2.12).

(2) From Theorem 4 in [10] and Proposition 2.5, the conclusion is direct.

\subsection{Sensitivity Analysis of Parameterized $P_{x}$}

In the following, we make sensitivity analysis of parameterized min-max programming $P_{x}$, that is, study variation of isolated local minimizers and corresponding marginal function under small perturbation of $x$.

For convenience of discussion, for any given parameter $x$, denote $y^{*}(x)$ as a minimizer of $P_{x}, v(x)=\min \{g(x, y): y \in Y\}$ as the corresponding marginal function value and make the following assumptions first.

Assumption 2.7. For given $x \in R^{n}$, the parametric problem $P_{x}$ is a convex problem, specifically, $g_{i}(x, y)$ and $i=1, \ldots, q$ are concave functions with respect to that variables $y$ and $h_{j}(y), j=$ $1, \ldots, l$ are convex functions.

Assumption 2.8. Let $I_{h}(y)=\left\{i \in L: h_{i}(y)=0\right\},\left\{\nabla h_{i}(y): i \in I_{h}(y)\right\}$ are linearly independent. 
Definition 2.9 (see Definition 2.1, [13]). For a given $\bar{x}, \bar{y} \in Y$ is said to be an isolated local minimum with order $i(i=1$ or 2$)$ of $P_{\bar{x}}$ if there exists a real $m>0$ and a neighborhood $V$ of $\bar{y}$ such that

$$
g(\bar{x}, y)>g(\bar{x}, \bar{y})+\frac{1}{2} m\|y-\bar{y}\|^{i}, \quad \forall y \in V \cap Y, y \neq \bar{y}
$$

Theorem 2.10. For a given $x \in R^{n}$, Assumptions 2.2-2.8 hold, then the following conclusions hold:

(1) if $y^{*}(x)$ with corresponding multiplier $\lambda^{*}$ is the solution of (2.3), then $y^{*}(x)$ is a unique first-order isolated minimizer of $P_{x}$;

(2) for any minimum $y^{*}(x)$, it is a locally Lipschitzian function with respect to $x$, that is, there exists a $L_{1}>0, \delta>0$ such that

$$
\left\|y^{*}\left(x^{k}\right)-y^{*}(x)\right\| \leq L_{1}\left\|x^{k}-x\right\|, \quad \forall x^{k} \in U(x, \delta), y^{*}\left(x^{k}\right) \in Y\left(x^{k}\right)
$$

where $Y\left(x^{k}\right)$ denotes minima set of $P_{x^{k}}$;

(3) for any minimum $y^{*}(x)$, marginal function $v(x)=g\left(x, y^{*}(x)\right)$ is also a locally Lipschitz function with respect to $x$, and $\partial v(x) \subseteq S(x)$, where

$$
S(x)=\operatorname{co}\left\{\nabla_{x} g_{i}\left(x, y^{*}(x)\right), i \in I\left(x, y^{*}(x)\right)\right\}
$$

and $I\left(x, y^{*}(x)\right)=\left\{i \in\{1, \ldots, q\}: g_{i}\left(x, y^{*}(x)\right)=g\left(x, y^{*}(x)\right)\right\}$. As a result,

$$
\partial v(x)=\left\{\sum_{i \in I\left(x, y^{*}(x)\right)} \lambda_{i} \nabla_{x} g_{i}\left(x, y^{*}(x)\right): \lambda_{i} \geq 0, \sum_{i \in I\left(x, y^{*}(x)\right)} \lambda_{i}=1\right\} .
$$

Proof. (1) From Assumption 2.7, it is direct that $y^{*}(x)$ is a global minimizer of $P_{x}$. We only prove $y^{*}(x)$ is a first-order isolated minimizer.

If the conclusion does not hold, then there exists a sequence $\left\{y^{k}\right\} \in Y(x)$ converging to $y^{*}(x), y^{k} \neq y^{*}(x)$, and a sequence $m_{k}, m_{k}>0$, and $m_{k}$ converges to 0 such that

$$
g\left(x, y^{k}\right) \leq g\left(x, y^{*}(x)\right)+\frac{1}{2} m_{k}\left\|y^{k}-y^{*}\right\|, \quad y^{k} \in Y
$$

Take $d_{k}=\left(y^{k}-y^{*}(x)\right) /\left\|y^{k}-y^{*}(x)\right\|$, for simplicity, we suppose $d_{k} \rightarrow d$, with $\|d\|=1$. Let $t_{k}=\left\|y^{k}-y^{*}(x)\right\|$, then from $y^{k} \in Y, d_{k} \rightarrow d$ and $Y$ is compact, we have

$$
y^{*}(x)+t_{k} d \in Y, \quad t_{k} \longrightarrow 0,
$$

that is,

$$
\nabla h_{i}\left(y^{*}(x)\right)^{T} d \leq 0, \quad \forall i \in I\left(x, y^{*}(x)\right)
$$


From Assumption 2.8, we know that $\sum_{i \in I\left(x, y^{*}(x)\right)} \nabla h_{i}\left(y^{*}(x)\right)^{T} d \neq 0$. As a result, we have $\sum_{i \in I\left(x, y^{*}(x)\right)} \nabla h_{i}\left(y^{*}(x)\right)^{T} d<0$.

From the first equation of (2.3), we know that there exists a $z \in \partial_{y} g\left(x, y^{*}(x)\right)$ such that for any feasible direction $d, z^{T} d=-\sum_{i \in I\left(x, y^{*}(x)\right)} \lambda_{i} \nabla h_{i}\left(y^{*}(x)\right)^{T} d>0$. Hence,

$$
g_{y}^{\prime}\left(x, y^{*}(x) ; d\right)=\max \left\{\xi^{T} d: \xi \in \partial_{y} g\left(x, y^{*}(x)\right)\right\} \geq z^{T} d>0
$$

On the other hand, from $y^{*}(x)$ is a minimizer, we know that $g_{y}^{\prime}\left(x, y^{*}(x) ; d\right) \geq 0$, this leads to a contradiction;

(2) from Assumption 2.8 and Theorem 3.1 in [13], the conclusion is direct;

(3) since $g(x, y)$ is a locally Lipschitzian function with respect to $x$ and $y$, then there exists $\delta>0, \delta^{\prime}>0$, and $L_{2}>0$ such that for any $x_{1} \in U(x, \delta), y \in U\left(y^{*}(x), \delta^{\prime}\right)$, it has

$$
\begin{gathered}
\left|g\left(x_{1}, y^{*}(x)\right)-g\left(x, y^{*}(x)\right)\right| \leq L_{2}\left\|x_{1}-x\right\| \\
\left|g(x, y)-g\left(x, y^{*}(x)\right)\right| \leq L_{2}\left\|y-y^{*}(x)\right\|
\end{gathered}
$$

As to $x_{1} \in U(x, \delta)$, from the conclusion in (1.2), there exists a a $L_{1}>0$ such that $\left\|y^{*}\left(x_{1}\right)-y^{*}(x)\right\| \leq L_{1}\left\|x_{1}-x\right\|$. As a result,

$$
\begin{aligned}
\mid v\left(x_{1}\right) & -v(x) \mid \\
& =\left|g\left(x_{1}, y^{*}\left(x_{1}\right)-g\left(x, y^{*}(x)\right)\right)\right| \\
& =\left|g\left(x_{1}, y^{*}\left(x_{1}\right)\right)-g\left(x_{1}, y^{*}(x)\right)+g\left(x_{1}, y^{*}(x)\right)-g\left(x, y^{*}(x)\right)\right| \\
& \leq\left|g\left(x_{1}, y^{*}\left(x_{1}\right)\right)-g\left(x_{1}, y^{*}(x)\right)\right|+\left|g\left(x_{1}, y^{*}(x)\right)-g\left(x, y^{*}(x)\right)\right| \\
& \leq L_{2}\left\|y^{*}\left(x_{1}\right)-y^{*}(x)\right\|+L_{2}\left\|x_{1}-x\right\| \leq L_{2}\left(1+L_{1}\right)\left\|x_{1}-x\right\| .
\end{aligned}
$$

Hence, the marginal function $v(x)$ is a local Lipschitzian function with respect to $x$.

Let $\widehat{S}(x)=\left\{-\nabla_{x} g_{i}(x, y(x)), i \in I(x, y(x))\right\}$, then $S(x)=\operatorname{co}\{\xi, \xi \in \widehat{S}(x)\}$. We prove that $\widehat{S}(x)$ is closed first, that is, prove for any sequence $\left\{x^{k}\right\} \subset R^{n}, x^{k} \rightarrow \bar{x}, z^{k} \in \widehat{S}\left(x^{k}\right), z^{k} \rightarrow z$, it has $z \in \widehat{S}(\bar{x})$.

From $z^{k} \in \widehat{S}\left(x^{k}\right)$, there exist $y^{k} \in Y\left(x^{k}\right) ; i_{k} \in I\left(x^{k}, y^{k}\right)$ such that $z^{k}=-\nabla_{x} g_{i_{k}}\left(x^{k}, y^{k}\right)$. Without loss of generality, suppose that $\left\{y^{k}\right\}$ converges to $\bar{y} ;\left\{i_{k}\right\}$ converges to $\bar{i}$. From Proposition 3.3 in [14], it has $\bar{y} \in Y(\bar{x})$, and $\bar{i} \in I(\bar{x}, \bar{y})$ and from $\nabla_{x} g_{\bar{i}}(x, y)$ is a continuous function, it has $z=\lim _{k \rightarrow+\infty} z_{k}=\lim _{k \rightarrow+\infty} \nabla_{x} g_{i_{k}}\left(x^{k}, y^{k}\right)=\nabla_{x} g_{\bar{i}}(\bar{x}, \bar{y}) \in \widehat{S}(x)$. As a result, $\widehat{S}(x)$ is a closed set. 
From Theorem 3.2.16 in [11], for any $\xi \in \partial v(x)$, there exists $x^{k} \in R^{n}, x^{k} \rightarrow x$ such that $\nabla v\left(x^{k}\right)$ exists and $\xi=\lim _{k \rightarrow+\infty} \nabla v\left(x^{k}\right)$. In addition, for arbitrary $d \in R^{n}$, it has

$$
\begin{aligned}
\nabla v\left(x^{k}\right)^{T} d & =v^{\prime}\left(x^{k} ; d\right)=\lim _{t \downarrow 0} \frac{v\left(x^{k}+t d\right)-v\left(x^{k}\right)}{t} \\
& =\lim _{t \downarrow 0} \frac{g\left(x^{k}+t d, y^{*}\left(x^{k}+t d\right)\right)-g\left(x^{k}, y^{*}\left(x^{k}\right)\right)}{t} \\
& \leq \lim _{t \downarrow 0} \frac{g\left(x^{k}+t d, y^{*}\left(x^{k}\right)\right)-g\left(x^{k}, y^{*}\left(x^{k}\right)\right)}{t} \\
& =\max _{i \in I\left(x^{k}, y\right)}\left\{-\nabla_{x} g_{i}\left(x^{k}, y\right)^{T} d\right\} .
\end{aligned}
$$

From the definition of $S\left(x^{k}\right), \exists z^{k} \in S\left(x^{k}\right)$ such that $z^{k^{T}} d=\max _{i \in I\left(x^{k}, y\right)}\left\{-\nabla_{x} g_{i}\left(x^{k}, y\right)^{T} d\right\}$. Hence, it has $\nabla v\left(x^{k}\right)^{T} d \leq z^{k^{T}} d$.

From $z^{k} \rightarrow z \in \widehat{S}(x) \subset S(x), \nabla v\left(x^{k}\right) \rightarrow \xi$ and $\nabla v\left(x^{k}\right)^{T} d \leq z^{k^{T}} d$, it has $\xi^{T} d \leq z^{T} d$, that is, for arbitrary $d \in R^{n}$ and $\xi \in \partial v(x)$, there exists $z \in S(x)$ such that $\xi^{T} d \leq z^{T} d$.

If $\partial v(x) \subset S(x)$ does not hold, then there exists a $\xi \in \partial v(x)$ and $\xi \notin S(x)$. From $S(x)$ is a compact convex set and separation theorem ([15]), there exists a $d \in R^{n}$ such that $\xi^{T} d<0$ and for arbitrary $z \in S(x), z^{T} d \geq 0$, which leads to a contradiction. As a result, $\partial v(x) \subset S(x)$ holds. From $\partial v(x) \subset S(x)$ and $S(x)=\operatorname{co}\left\{\nabla_{x} g_{i}\left(x, y^{*}(x)\right), i \in I\left(x, y^{*}(x)\right)\right\}$, computation formula (2.17) is direct.

\section{Discussion}

In this paper, sufficient optimality and sensitivity analysis of a parameterized min-max programming are given. A rule for computation the subdifferential of $v(x)$ is established. Though the assumptions in this paper are some restrictive compared to some existing work, the assumptions hold naturally for some applications. Moreover, the obtained computation formula is simple, it is beneficial for establishing a concise first-order necessary optimality system of (1.1), and then constructing effective algorithms to solve the applications.

\section{Acknowledgments}

This research was supported by the National Natural Science Foundation of China no. 11001092 and the Fundamental Research Funds for the Central Universities no. 2011QC064.

\section{References}

[1] C. Kirjner-Neto and E. Polak, "On the conversion of optimization problems with max-min constraints to standard optimization problems," SIAM Journal on Optimization, vol. 8, no. 4, pp. 887-915, 1998.

[2] E. Polak and J. O. Royset, "Algorithms for finite and semi-infinite min-max-min problems using adaptive smoothing techniques," Journal of Optimization Theory and Applications, vol. 119, no. 3, pp. 421-457, 2003. 
[3] G.-X. Liu, "A homotopy interior point method for semi-infinite programming problems," Journal of Global Optimization, vol. 37, no. 4, pp. 631-646, 2007.

[4] O. Stein and G. Still, "Solving semi-infinite optimization problems with interior point techniques," SIAM Journal on Control and Optimization, vol. 42, no. 3, pp. 769-788, 2003.

[5] O. Stein and A. Tezel, "The semismooth approach for semi-infinite programming under the reduction ansatz," Journal of Global Optimization, vol. 41, no. 2, pp. 245-266, 2008.

[6] A. Auslender, "Stability in mathematical programming with nondifferentiable data," SIAM Journal on Control and Optimization, vol. 22, no. 2, pp. 239-254, 1984.

[7] J. F. Bonnans and A. Shapiro, Perturbation Analysis of Optimization Problems, Springer, New York, NY, USA, 2000.

[8] J. F. Bonnans and A. Shapiro, "Optimization problems with perturbations: a guided tour," SIAM Review, vol. 40, no. 2, pp. 228-264, 1998.

[9] B. S. Mordukhovich, N. M. Nam, and N. D. Yen, "Subgradients of marginal functions in parametric mathematical programming," Mathematical Programming B, vol. 116, no. 1-2, pp. 369-396, 2009.

[10] R. W. Chaney, "Second-order sufficient conditions in nonsmooth optimization," Mathematics of Operations Research, vol. 13, no. 4, pp. 660-673, 1988.

[11] M. M. Mäkelä and P. Neittaanmäki, Nonsmooth Optimizatin: Analysis and Algorithms with Application to Optimal Control, Utopia Press, Singapore, 1992.

[12] L. Huang and K. F. Ng, "Second-order optimality conditions for minimizing a max-function," Science in China A, vol. 43, no. 7, pp. 722-733, 2000.

[13] B. S. Mordukhovich, "Sensitivity analysis in nonsmooth optimization," in Theoretical Aspects of Industrial Design, D. A. Field and V. Komkov, Eds., pp. 32-46, SIAM, Philadelphia, Pa, USA, 1992.

[14] V. F. Demyanov and A. M. Rubinov, Constructive Nonsmooth Analysis, vol. 7, Peter Lang, Frankfurt am Main, Germany, 1995.

[15] R. T. Rochafellar, Convex Analysis, Princeton University Press, Princeton, NJ, USA, 1970. 


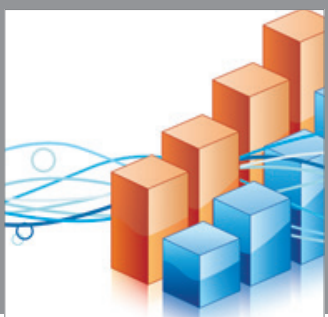

Advances in

Operations Research

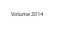

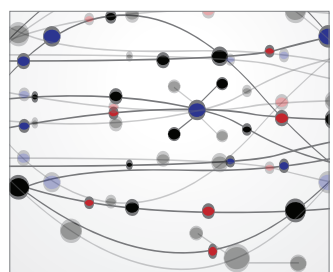

\section{The Scientific} World Journal
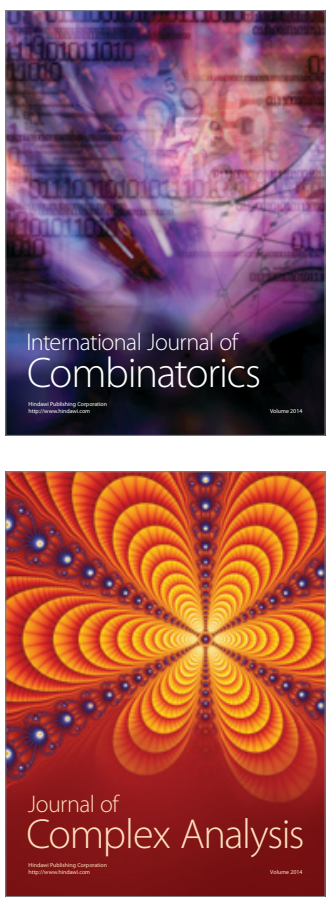

International Journal of

Mathematics and

Mathematical

Sciences
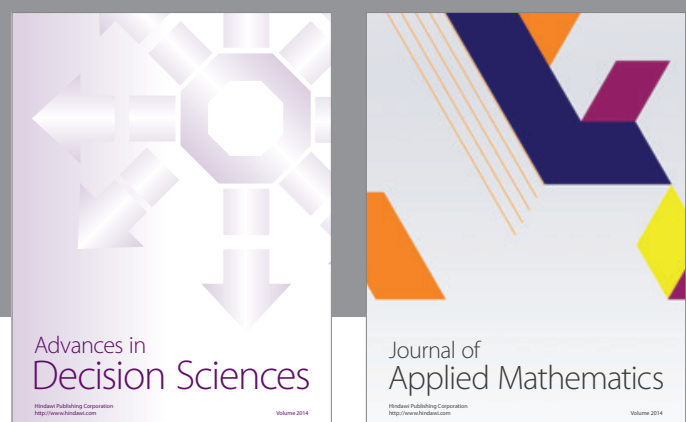

Journal of

Applied Mathematics
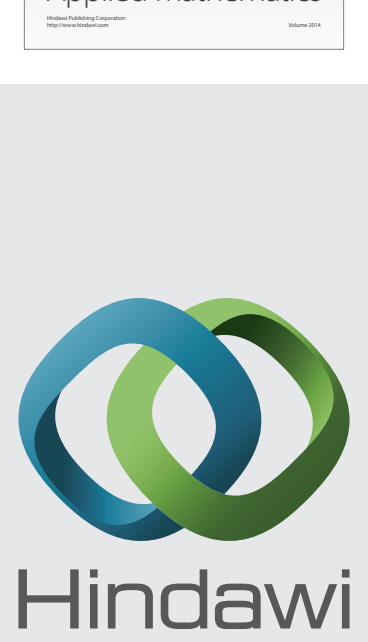

Submit your manuscripts at http://www.hindawi.com
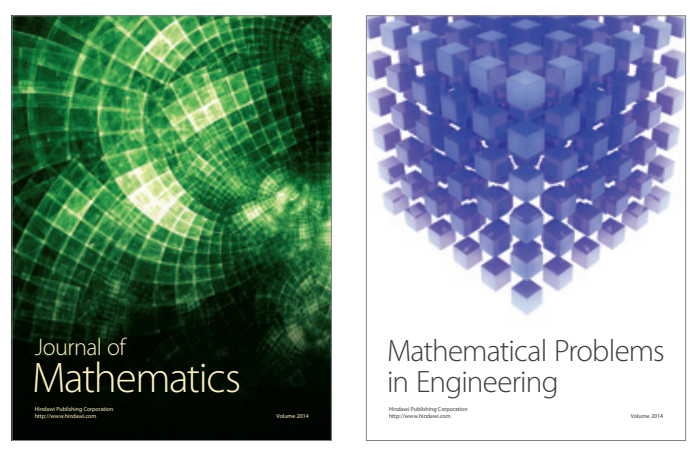

Mathematical Problems in Engineering
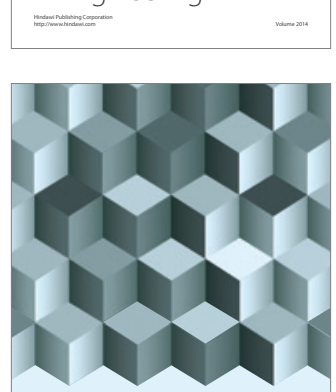

Journal of

Function Spaces
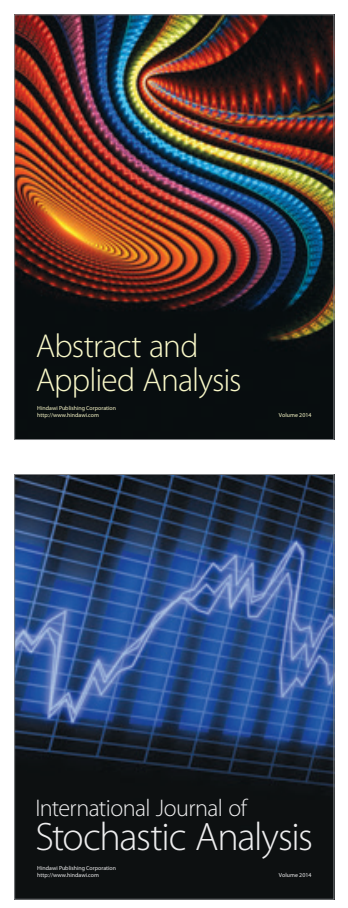

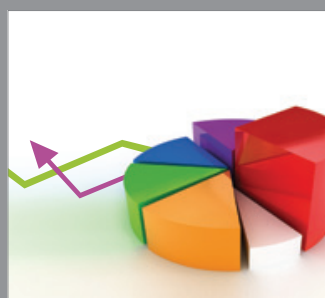

ournal of

Probability and Statistics

Promensencen
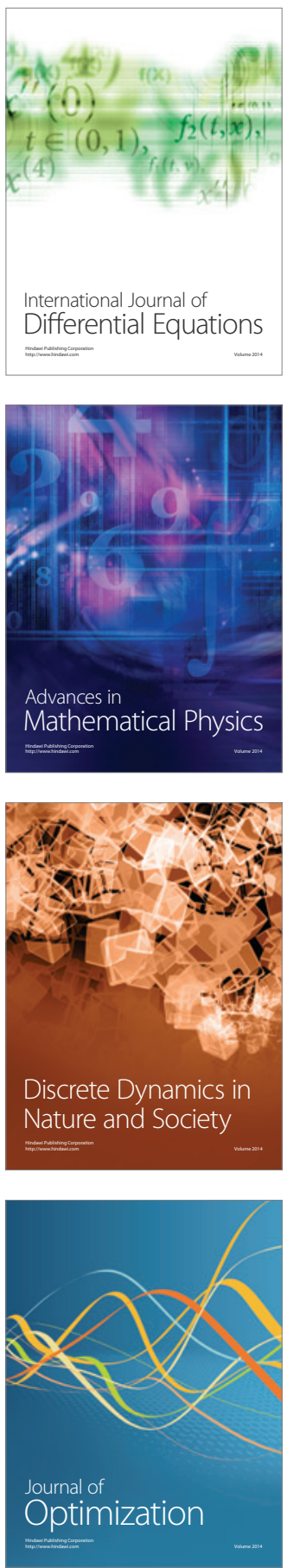